\title{
Prophylaxis of breast cancer chemotherapy for bone marrow toxicity: more individualized and safer
}

\author{
Fengrui Xu, Zefei Jiang \\ Department of Breast Oncology, The Fifth Medical Center of Chinese PLA General Hospital, Beijing 100071, China \\ Correspondence to: Zefei Jiang. Department of Breast Oncology, The Fifth Medical Center of Chinese PLA General Hospital, No. 8 East Street, \\ Fengtai District, Beijing 100071, China. Email: jiangzefei@csco.org.cn. \\ Provenance: This is an invited article commissioned by the Editorial Office of Annals of Translational Medicine. \\ Response to: Tirelli U, Carbone A, Di Francia R, et al. A new peg-filgrastim biosimilar, mecapegfilgrastim for primary prophylaxis of chemotherapy- \\ related neutropenia is now available. Ann Transl Med 2019. doi: 10.21037/atm.2019.10.61
}

Submitted Nov 20, 2019. Accepted for publication Dec 05, 2019.

doi: $10.21037 / \mathrm{atm} .2019 .12 .50$

View this article at: http://dx.doi.org/10.21037/atm.2019.12.50

We thank Dr. Tirelli, Dr. Carbone, Dr. Francia and Dr. Di Francia for the interest they have taken in our paper "Efficacy and safety of mecapegfilgrastim for prophylaxis of chemotherapy-induced neutropenia in patients with breast cancer: a randomized, multicenter, active-controlled phase III trial", and appreciate their comments $(1,2)$.

In our phase III study, Long-acting mecapegfilgrastim $(100 \mu \mathrm{g} / \mathrm{kg}$ and fixed $6 \mathrm{mg})$ is effective and well tolerated when administered in the primary prophylaxis of chemotherapy induced neutropenia and in consecutivecycle treatment. The fixed $6 \mathrm{mg}$-dose regimen showed similar efficacy and safety profile compared with $100 \mu \mathrm{g} / \mathrm{kg}$ regimen, and would be the preference in clinical practice due to the convenient once-per-cycle administration and high-degree treatment compliance for the patients.

High costs of cancer care place considerable burden on patients and society. It is increasingly recognized provider should play a role in reducing the cost of care (3). Our previous research shows that the economic factors of the drug influence the choice of treatment and may affect the patient's benefit (4). Compared with filgrastim, the duration of grade 4 neutropenia was significantly shorter in patients with mecapegfilgrastim. The reduced duration of severe neutropenia (DSN) could be associated with decreased risk of infection and shorter period of hospitalization, which may be related to decreased cost. Now the mecapegfilgrastim has been licensed by National Medical Products Administration of China, and the pharmacoeconomic research would be one of our research plans to investigate more details about the cost saving when mecapegfilgrastim used.
As Dr. Tirelli and co-authors have said, in addition to the enrolled population, the new long-acting mecapegfilgrastim may be effective in some frail subgroups of patients like elderly patients and HIV-related tumors. Some studies have shown that patients over 65 are likely to experience more serious adverse events when exposed to same dose as young patients (5). HIV patients may be more susceptible than non-HIV patients to infectious complications following chemotherapy. A very early study showed that G-CSF significantly improved dose-intensity of chemotherapy for patients with HIV-related NHL (6).

In fact, we have carried out real-world studies (RWS) that have included patients of all ages, and we have further explored the protective effects in patients with excessive doses of chemotherapy that can be seen in the real world. Preliminary results show that mecapegfilgrastim has the same protective effect on elderly patients, and despite the protection of drugs, excessive doses of chemotherapy still bring more serious bone marrow toxicity. It is suggested that in clinical practice, even if the PEG-rhg-CSF is used prophylactically, a standard dose chemotherapy should be used.

Although the advantages of long-acting mecapegfilgrastim were observed in this study, it was also found that even with the prophylactic application of mecapegfilgrastim, about $30 \%$ of patients still experienced grade 4 neutropenia in the first cycle of chemotherapy. Finding these patients in advance and optimizing protection measures are the next important work. With the development of artificial intelligence, we plan to use the method of machine learning and combine the data 
both from the clinical research and real-world application of mecapegfilgrastim, hoping to establish a prediction model of bone marrow toxicity of breast cancer chemotherapy to promote individualized chemotherapy management.

\section{Acknowledgments}

None.

\section{Footnote}

Conflicts of Interest: The authors have no conflicts of interest to declare.

Ethical Statement: The authors are accountable for all aspects of the work in ensuring that questions related to the accuracy or integrity of any part of the work are appropriately investigated and resolved.

\section{References}

1. Tirelli U, Carbone A, Di Francia R, et al. A new pegfilgrastim biosimilar, mecapegfilgrastim for primary prophylaxis of chemotherapy-related neutropenia is now available. Ann Transl Med 2019. doi: 10.21037/

Cite this article as: Xu F, Jiang Z. Prophylaxis of breast cancer chemotherapy for bone marrow toxicity: more individualized and safer. Ann Transl Med 2020;8(4):138. doi: 10.21037/ atm.2019.12.50 atm.2019.10.61.

2. Xu F, Zhang Y, Miao Z, et al. Efficacy and safety of mecapegfilgrastim for prophylaxis of chemotherapyinduced neutropenia in patients with breast cancer: a randomized, multicenter, active-controlled phase III trial. Ann Transl Med 2019;7:482.

3. Resnicow K, Patel MR, Mcleod MC, et al. Physician attitudes about cost consciousness for breast cancer treatment: differences by cancer sub-specialty. Breast Cancer Res Treat 2019;173:31-6.

4. Li J, Wang S, Wang Y, et al. Disparities of Trastuzumab Use in Resource-Limited or Resource-Abundant Regions and Its Survival Benefit on HER2 Positive Breast Cancer: A Real-World Study from China. Oncologist 2017;22:1333-8.

5. Lichtman SM, Hurria A, Cirrincione CT, et al. Paclitaxel efficacy and toxicity in older women with metastatic breast cancer: combined analysis of CALGB 9342 and 9840 . Ann Oncol 2012;23:632-8.

6. Rossi G, Donisi A, Casari S, et al. Effects of recombinant granulocyte colony-stimulating factor (G-CSF) in patients treated with ProMACE-CytaBOM for HIVrelated non-Hodgkin's lymphoma (NHL). Haematologica 1998;83:317-22. 